below the analytic method's limit of detection (LOD), making estimation of exposure levels challenging. The concentration of each chemical relative to THC is related to the concentration of the chemical and THC in the source crude oil. Knowing these relationships, we can develop models to predict concentrations of individual chemicals from THC concentrations when only a THC concentration was detectable. The goal of this study was to determine the correlations between concentrations of the various oil chemicals and THC for use in situations where only THC was above the LOD.

Method We calculated correlations on the rig ships and support vessels located near the well by vessel and time period using linear regression analysis that accounts for censored data.

Results We found significant differences in correlations between concentrations of the chemicals and THC across vessels and over time that likely reflect different vessel activities and degrees of crude oil weathering throughout the response and clean-up efforts.

Conclusions Correlations between concentrations of the chemicals of interest and THC can be used to estimate the chemical's concentration when its measurement is below the LOD.

\section{THE PREVALENCE OF RISK FACTORS ASSOCIATED WITH COMPUTER VISION SYNDROME AMONG COMPUTER WORKS IN SÃO PAULO, BRAZIL}

Eduardo Sá, Lys Rocha. Faculdade de Medicina Da Universidade de São Paulo, São Paulo, Brazil

\subsection{6/oemed-2014-102362.322}

Objectives The aims of this study were to investigate work conditions, to estimate the prevalence and to describe risk factors associated with Computer Vision Syndrome among computer works in São Paulo.

Method The methods include a quantitative cross-sectional observational study and an ergonomic work analysis, using work observation, interviews and questionnaires. The case definition was the presence of one or more specific ocular symptoms answered as always, often or sometimes. The multiple logistic regression model, were created using the stepwise forward likelihood method and remained the variables with levels below 5\% $(\mathrm{p}<0.05)$.

Results The operators were mainly female and young (from 15 to 24 years old). The call centre was opened $24 \mathrm{~h}$ and the operators weekly hours were $36 \mathrm{~h}$ with break time from 21 to $35 \mathrm{~min}$ per day. The symptoms reported were eye fatigue (73.9\%), "weight" in the eyes (68.2\%), "burning" eyes (54.6\%), tearing (43.9\%) and weakening of vision (43.5\%). The prevalence of Computer Vision Syndrome was 54.6\%.

Conclusions The organisation and psychosocial factors at work should be included in prevention programs of visual syndrome among call centres' operators.

\section{TECHNICAL EPIDEMIOLOGICAL NEXUS (NTEP) IN BRAZIL: A CRITIC EVALUATION AFTER 5 YEARS OF ITS APPLICATION}

Eduardo Sá, Miguel Fernandes. Faculdade de Medicina Da Universidade de São Paulo, São Paulo, Brazil

\subsection{6/oemed-2014-102362.323}

Objectives A study has been made regarding the 5 years of Technical Epidemiological Nexus' application, analysing the amounts collected, the number of occupational accidents recognised in the period, the benefits granted and the main diseases recognised as accidentary.

Method It has been performed a bibliographic review of the literature, a study of chapters from books, scientific articles published and research on the website of the Social Providence, evaluating the statistic data from 2007 to 2011 and comparing with the last years without the new previdenciary legislation.

Results From the application in NTEP for the recognition of accidents and aggravations to workers' health, the values collected by INSS increased progressively, with an increase of $14.8 \%$ in 9 months of 2007 . The number of benefits granted rose 4,3\%, from 4 million in 2006 to 4.8 million in 2011. With NTEP there has been an increase in the number of pathologies related to occupation recognised by INSS. Of the 653.1 thousands accidents recognised in the year of 2007, 131.6 thousands $(20,15 \%)$ were recognised by the new rule, without the need of occupational accident communication. The pathologies of the spine (CID M54) and the ones of the shoulder (CID M75) are among the ones of highest incidence as occupational pathologies. Conclusions In the five years applying the technical epidemiological nexus there were increases in levy of the INSS from the number of benefits granted and the recognition of occupational accidents, the pathologies of the shoulder and spine being the highest ones to increase after the new rule.

\section{THE NIEHS GULF STUDY: RECALCULATION OF EXPOSURE MEASUREMENT DATA BETWEEN THE LIMIT OF DETECTION (LOD) REPORTED BY THE LABORATORY AND THE ANALYTICAL METHODS' LODS}

${ }^{1}$ Mark Stenzel, ${ }^{2}$ Patricia Stewart, ${ }^{3}$ Aaron Blair, ${ }^{4}$ Lawrence Engel, ${ }^{4}$ Richard Kwok, ${ }^{4}$ Dale Sandler. ${ }^{1}$ Exposure Assessment Applications, LLC, Arlington, VA, USA; ${ }^{2}$ Stewart Exposure Assessments, LLC, Arlington, VA, USA; ${ }^{3}$ National Cancer Institute, Gaithersburg, MD, USA; ${ }^{4}$ Epidemiology Branch, National Institute of Environmental Health Sciences, Research Triangle Park, NC, USA

\subsection{6/oemed-2014-102362.324}

Objectives BP contractors collected nearly 25000 personal passive dosimeter samples (about 150000 individual exposure measurements, primarily benzene, ethylbenzene, toluene, xylene and total hydrocarbon (THC)), related to the response and cleanup of the Deepwater Horizon oil spill. BP used a sampling strategy based on compliance with applicable occupational exposure limits (OELs). Most of the measurements were below the reported limit of detection (censored). This occurred because the analytic laboratories calibrated their instruments relative to the chemicals' OELs and they reported measurements below the lowest calibration standard (approximately 5\% of the OEL) as less than the LOD. In an epidemiology study, however, all exposure levels are of interest rather than only those levels related to an OEL. Published evaluation studies on the analytical methods indicate that the methods were capable of measuring much lower concentrations than those reported. This presentation discusses the process used to recalculate the measurement data to the analytic method's LOD.

Method Gas chromatograph output, the dosimeters' and chemicals' physical property data, and the slope and intercept of calibration curves were used to calculate concentrations below reported LODs.

Results The recalculation effort resulted in the reduction of all censored measurements from $92.8 \%$ to $60.2 \%$ and the THC censored measurements from $71.9 \%$ to $19.1 \%$. 